Portland State University

PDXScholar

$12-1-2011$

\title{
Nonlinear Ordinary Differential Equations in Fluid Dynamics
}

John D. Ramshaw

Portland State University, jdramshaw@yahoo.com

Follow this and additional works at: https://pdxscholar.library.pdx.edu/phy_fac

Part of the Physics Commons

Let us know how access to this document benefits you.

Citation Details

Ramshaw, J. D. (2011). Nonlinear ordinary differential equations in fluid dynamics. American Journal Of Physics, 79(12), 1255-1260.

This Article is brought to you for free and open access. It has been accepted for inclusion in Physics Faculty Publications and Presentations by an authorized administrator of PDXScholar. Please contact us if we can make this document more accessible: pdxscholar@pdx.edu. 


\title{
Nonlinear ordinary differential equations in fluid dynamics
}

\author{
John D. Ramshaw \\ Department of Physics, Portland State University, Portland, Oregon 97207
}

(Received 9 June 2011; accepted 18 August 2011)

\begin{abstract}
The equivalence between nonlinear ordinary differential equations (ODEs) and linear partial differential equations (PDEs) was recently revisited by Smith, who used the equivalence to transform the ODEs of Newtonian dynamics into equivalent PDEs, from which analytical solutions to several simple dynamical problems were derived. We show how this equivalence can be used to derive a variety of exact solutions to the PDEs describing advection in fluid dynamics in terms of solutions to the equivalent ODEs for the trajectories of Lagrangian fluid particles. The PDEs that we consider describe the time evolution of non-diffusive scalars, conserved densities, and Lagrangian surfaces advected by an arbitrary compressible fluid velocity field $\mathbf{u}(\mathbf{x}, t)$. By virtue of their arbitrary initial conditions, the analytical solutions are asymmetric and three-dimensional even when the velocity field is one-dimensional or symmetrical. Such solutions are useful for verifying multidimensional numerical algorithms and computer codes for simulating advection and interfacial dynamics in fluids. Illustrative examples are discussed. @ 2011 American Association of Physics Teachers.
\end{abstract}

[DOI: $10.1119 / 1.3636635]$

\section{INTRODUCTION}

The relation between nonlinear ordinary differential equations (ODEs) and linear (or more generally quasi-linear) first-order partial differential equations (PDEs) with variable coefficients is a standard topic in textbooks on PDEs. Most treatments begin with the PDEs and derive the corresponding ODEs, which is natural from a PDE perspective. In many physical applications, it is more straightforward to begin with the nonlinear ODEs and derive the corresponding PDEs, as was done by Carathéodory. ${ }^{1}$ The latter approach is natural from an ODE perspective but is rarely found in textbooks on ODEs, perhaps because it presumes a familiarity with PDEs that beginning students of ODEs have not yet acquired.

The equivalence between ODEs and PDEs, and the techniques for transforming from one to the other, are useful in several branches of physics, including classical mechanics, statistical physics, and fluid dynamics. A knowledge of those techniques deserves to be in the repertoire of all practicing physicists and engineers. Their applicability to classical dynamics has recently been discussed by Smith, ${ }^{2}$ who transformed the ODEs of Newtonian dynamics into equivalent PDEs, from which the analytical solutions to several basic dynamical problems were derived. In this paper, we exploit the same equivalence but in the reverse direction. We construct solutions of certain PDEs occurring in fluid dynamics in terms of solutions to the corresponding ODEs that describe the trajectories of Lagrangian fluid particles. The present development complements that of Smith, and illustrates that the equivalence between ODEs and PDEs may be useful in transforming the equations in either direction depending on the circumstances.

The PDEs that we consider describe the time evolution of non-diffusive scalars, conserved densities, and Lagrangian surfaces advected by an arbitrary compressible fluid velocity field $\mathbf{u}(\mathbf{x}, t)$, which is presumed to be given or known. Of course, the velocity field is not normally known in practical fluid dynamics problems, which are rarely tractable analytically and must usually be solved by numerical methods. ${ }^{3}$ However, analytical solutions of the present type are very useful in verifying the accuracy of the numerical methods and computer codes that are used to solve such problems. Their utility is greatly enhanced by the fact that the resulting analytical solutions are the general solutions of the PDEs, which are valid for arbitrary initial conditions that need not share the symmetry or simplicity of the velocity field. These solutions are consequently asymmetric and fully threedimensional even when the velocity field is one-dimensional or symmetrical. Such solutions are especially valuable for verifying three-dimensional numerical methods and computer codes, because most other known exact solutions in fluid dynamics are restricted to simple situations in which symmetries reduce the problem to one or two spatial dimensions. We emphasize, however, that our primary purpose is not to provide exact solutions for computational applications, but rather to foster a greater awareness of the mathematical methods involved by showing, in the context of fluid dynamics, how the relations discussed by $\mathrm{Smith}^{2}$ may be used to construct solutions to PDEs from those of the corresponding ODEs.

The relations upon which the present development is based are not new, but they are not widely appreciated in the fluid dynamics community. This situation may be due to the fact that those relations inherently involve the trajectories of Lagrangian fluid particles, which define the transformations between Lagrangian and Eulerian coordinates. These transformations are familiar in solid and continuum mechanics ${ }^{4}$ but play only a minor role in fluid dynamics. The reason is that the Eulerian form of the governing equations is usually much more convenient to work with (except in one spatial dimension). Consequently, Lagrangian coordinates receive only passing mention in most textbooks and courses on fluid dynamics, in spite of the fact that Lagrangian trajectories and the coordinate transformations they define are the very essence of kinematics, and as such are fundamental to an understanding of advection.

The general relations developed in this paper are formally valid for an arbitrary compressible velocity field $\mathbf{u}(\mathbf{x}, t)$, but are useful only when the latter is sufficiently simple that the ODE's that determine the motion of the Lagrangian fluid 
particles are integrable, so that the trajectories can be expressed in closed form. In practice, integrability usually entails a restriction to separable velocity fields of the form $\mathbf{u}(\mathbf{x}, t)=\mathbf{U}(\mathbf{x}) T(t)$. The implications and simplifications that result from this restriction will be discussed in detail.

As a simple example of the type of results that can be derived using the relations developed in this paper, consider the problem of solving the one-dimensional continuity equation

$$
\frac{\partial \rho}{\partial t}+\frac{\partial(\rho u)}{\partial x}=0
$$

where $u$ is a given time-independent velocity field of the form $u(x, t)=x / T$, and $\rho(x, t)$ is the unknown fluid density which evolves from the initial condition

$$
\rho(x, 0)=\frac{A}{1+(x / L)^{2}} .
$$

Here $x$ is a Cartesian spatial coordinate, $t$ is the time, and $L$, $T$, and $A$ are constants with units of length, time, and density, respectively. This problem has the analytical solution

$$
\rho(x, t)=\frac{A e^{-t / T}}{1+(x / L)^{2} e^{-2 t / T}},
$$

as can be verified by direct substitution. Readers might find it interesting, and possibly challenging, to directly derive this solution using only the standard techniques of calculus, which requires some ingenuity. However, the same solution is easily obtained in just a few lines, requiring no ingenuity whatsoever, as a special case of the much more general Eq. (20), which merely requires one to solve for the fluid particle trajectories defined by $d x / d t=x / T$.

\section{LAGRANGIAN TRAJECTORIES}

The present development is based on the trajectories of Lagrangian fluid particles, which satisfy the ordinary differential equation

$$
\dot{\mathbf{x}}=\mathbf{u}(\mathbf{x}, t),
$$

where $\mathbf{x}$ is the position vector of the particle in threedimensional space at time $t$. The formal solution of Eq. (4) is denoted by

$$
\mathbf{x}=\mathbf{F}(t ; \mathbf{X}),
$$

where $\mathbf{X}$ is the initial position of the particle at $t=0$; that is, the Lagrangian coordinates of the particle. Clearly, $\mathbf{F}(0 ; \mathbf{X})=\mathbf{X}$ and $\partial \mathbf{F} / \partial t=\mathbf{u}(\mathbf{F}, t)$. In principle, Eq. (5) may be solved for $\mathbf{X}$ to obtain

$$
\mathbf{X}=\mathbf{G}(t ; \mathbf{x}),
$$

and clearly $\mathbf{G}(0 ; \mathbf{x})=\mathbf{x}$. Equation (6) defines the transformation from Eulerian to Lagrangian coordinates, and Eq. (5) defines the reverse transformation.

By differentiating Eq. (6) with respect to time and using Eq. (4), we find that $\mathbf{G}(t ; \mathbf{x})$ satisfies a first-order linear homogeneous PDE with variable coefficients:

$$
\frac{\partial \mathbf{G}}{\partial t}+\mathbf{u} \cdot \nabla \mathbf{G}=0
$$

where $\nabla \equiv \partial / \partial \mathbf{x}$. The nonlinear ODE of Eq. (4) is therefore equivalent to the linear PDE of Eq. (7), and vice versa. Equation (7) is sometimes useful for formal purposes, but rarely for directly determining $\mathbf{G}$. Even though it is linear, the fact that it contains variable coefficients precludes its analytical solution except in simple special cases.

It is sometimes of interest to determine the velocity field that will produce given Lagrangian trajectories. Because the latter uniquely determine the functions $\mathbf{F}(t ; \mathbf{X})$ and $\mathbf{G}(t ; \mathbf{x})$, the velocity field may be determined by combining Eqs. (4) and (5) to obtain $\mathbf{u}(\mathbf{x}, t)=\partial \mathbf{F}(t ; \mathbf{X}) / \partial t$ and replacing $\mathbf{X}$ therein by $\mathbf{G}(t ; \mathbf{x})$ in accordance with Eq. (6).

\section{SEPARABLE VELOCITY FIELDS}

The problem of solving Eq. (4) is greatly simplified if the velocity field is separable in the sense that $\mathbf{u}(\mathbf{x}, t)=\mathbf{U}(\mathbf{x}) T(t)$. In this case, it is convenient to define the function $\tau(t)$ by

$$
\frac{d \tau}{d t}=T(t)
$$

subject to the initial condition $\tau(0)=0$. We may then transform from $t$ to $\tau$ as an independent variable, so that Eq. (4) takes the form

$$
\frac{d \mathbf{x}}{d \tau}=\mathbf{U}(\mathbf{x})
$$

which in contrast to Eq. (4) is now an autonomous ordinary differential equation for $\mathbf{x}$. The formal solution of Eq. (9) subject to the initial condition that $\mathbf{x}=\mathbf{X}$ at $\tau=0$ may be written as

$$
\mathbf{x}=\mathbf{F}_{s}(\tau ; \mathbf{X}),
$$

where $\mathbf{F}_{s}(0 ; \mathbf{X})=\mathbf{X}$, and the subscript $s$ reminds us of the separability and the fact that $\mathbf{x}$ is the solution of the associated autonomous Eq. (9), and hence depends on $\tau$ rather than $t$. But $\mathbf{F}_{s}$ is sufficient to determine the full solution of Eq. (4) in this context, because comparison with Eq. (5) shows that $\mathbf{F}(t ; \mathbf{X})=\mathbf{F}_{s}(\tau(t) ; \mathbf{X})$.

As before, Eq. (10) may in principle be solved for $\mathbf{X}$ to obtain

$$
\mathbf{X}=\mathbf{G}_{s}(\tau ; \mathbf{x}),
$$

and clearly $\mathbf{G}_{s}(0 ; \mathbf{x})=\mathbf{x}$. In contrast to the general nonautonomous case, there is now a much simpler way to obtain $\mathbf{G}_{s}$ from $\mathbf{F}_{s}$. Autonomy implies that $\tau$ is an affine variable; that is, only differences between $\tau$ values are significant, which can be formally shown by transforming to the new variable $\tau^{\prime}=\tau-\tau_{1}$, where $\tau_{1}$ is an arbitrary reference value of $\tau$. Equation (9) then takes the form $d \mathbf{x} / d \tau^{\prime}=\mathbf{U}(\mathbf{x})$, which has the same form as Eq. (9) with $\tau$ replaced by $\tau^{\prime}$. It follows that $\mathbf{x}=\mathbf{F}_{s}\left(\tau^{\prime} ; \mathbf{X}^{\prime}\right)$, where $\mathbf{X}^{\prime}$ is the value of $\mathbf{x}$ at $\tau^{\prime}=0$; that is, the value of $\mathbf{x}$ at $\tau=\tau_{1}$. Therefore, $\mathbf{x}(\tau)=\mathbf{F}_{s}\left(\tau-\tau_{1} ; \mathbf{x}\left(\tau_{1}\right)\right)$. But $\mathbf{x}(\tau)$ is given by Eq. (10) as well, so that $\mathbf{F}_{s}(\tau ; \mathbf{X})$ $=\mathbf{F}_{s}\left(\tau-\tau_{1} ; \mathbf{x}\left(\tau_{1}\right)\right)$. We set $\tau=0$ and obtain $\mathbf{F}_{s}\left(-\tau_{1} ; \mathbf{x}\left(\tau_{1}\right)\right)$ $=\mathbf{F}_{s}(0 ; \mathbf{X})=\mathbf{X}$. Because $\tau_{1}$ is a dummy variable, we may replace it by $\tau$ to obtain $\mathbf{X}=\mathbf{F}_{s}(-\tau ; \mathbf{x})$, which simply states that in an autonomous system, the roles of the initial and 
final points $\mathbf{X}$ and $\mathbf{x}$ may be interchanged by running backward in $\tau$. Comparison with Eq. (11) shows that

$$
\mathbf{G}_{s}(\tau ; \mathbf{x})=\mathbf{F}_{s}(-\tau ; \mathbf{x}),
$$

which immediately determines $\mathbf{G}_{s}$ from $\mathbf{F}_{s}$ without any functional inversion. If we combine Eqs. (11) and (12) and compare with Eq. (6), we obtain $\mathbf{G}(t ; \mathbf{x})=\mathbf{F}_{s}(-\tau(t) ; \mathbf{x})$, so that determination of $\mathbf{F}_{s}$ immediately determines $\mathbf{G}$ as well.

As before, we readily verify that $\mathbf{G}_{s}(\tau ; \mathbf{x})$ satisfies the equation

$$
\frac{\partial \mathbf{G}_{s}}{\partial \tau}+\mathbf{U} \cdot \nabla \mathbf{G}_{s}=0
$$

which combines with Eq. (12) to show that $\mathbf{F}_{s}(\tau ; \mathbf{X})$ satisfies the conjugate equation

$$
\frac{\partial \mathbf{F}_{s}}{\partial \tau}-\mathbf{U}(\mathbf{X}) \cdot \frac{\partial \mathbf{F}_{s}}{\partial \mathbf{X}}=0,
$$

for which there is no simple analog in the nonautonomous case. Because $\mathbf{U}$ is independent of $\tau$, Eqs. (13) and (14) can be formally solved in terms of exponential operators: $\mathbf{G}_{s}(\tau ; \mathbf{x})=\exp \{-\tau \mathcal{L}(\mathbf{x})\} \mathbf{x} \quad$ and $\quad \mathbf{F}_{s}(\tau ; \mathbf{X})=\exp \{\tau \mathcal{L}(\mathbf{X})\} \mathbf{X}$, where $\mathcal{L}(\mathbf{x}) \equiv \mathbf{U}(\mathbf{x}) \cdot \nabla$. Equations (13) and (14), and their formal solutions given previously, are sometimes useful theoretically, but rarely for actually determining $\mathbf{F}_{s}$ and $\mathbf{G}_{s}$.

Although the restriction to separable velocity fields introduces the simplifications associated with autonomy, the analytical solution of Eq. (9) is possible only in exceptional cases. Even when $\mathbf{U}(\mathbf{x})$ is smooth and simple in form, the solution $\mathbf{F}_{s}(\tau ; \mathbf{X})$ may be singular and hopelessly complicated, as illustrated by the Lorenz equations and other chaotic systems with strange attractors.

\section{NON-DIFFUSIVE SCALARS}

A non-diffusive scalar is a scalar quantity $f$ whose value for any Lagrangian fluid particle is constant in time, so that

$$
f(\mathbf{x}, t)=f(\mathbf{X}, 0) \equiv f_{0}(\mathbf{X}) .
$$

It follows immediately from Eqs. (6) and (15) that

$$
f(\mathbf{x}, t)=f_{0}(\mathbf{G}(t ; \mathbf{x})),
$$

which expresses the function $f(\mathbf{x}, t)$ in terms of its initial value $f_{0}(\mathbf{x})$, provided that the function $\mathbf{G}(t ; \mathbf{x})$ is known.

By differentiating Eq. (15) with respect to time at constant $\mathbf{X}$ and making use of Eq. (4), we find that $f(\mathbf{x}, t)$ satisfies the PDE

$$
\frac{\partial f}{\partial t}+\mathbf{u} \cdot \nabla f=0
$$

which is frequently adopted as an alternative equivalent definition of a non-diffusive scalar. Equation (16) shows that the solution of this PDE subject to the initial condition $f(\mathbf{x}, 0)=f_{0}(\mathbf{x})$ can be expressed in terms of the solution to the ODE of Eq. (4) as a function of $\mathbf{X}$. This equivalence reflects the fact that the hyperbolic PDE of Eq. (17) can, in principle, be solved by the method of characteristics, ${ }^{5}$ which in the present context are the fluid particle trajectories determined by Eq. (4).
Note that all non-diffusive scalars satisfy the same PDE, namely Eq. (17), so that different non-diffusive scalars differ only in their initial conditions. It is also easy to verify that any function of a non-diffusive scalar is another nondiffusive scalar. A comparison between Eqs. (7) and (17) shows that $\mathbf{G}(t ; \mathbf{x})$ satisfies the same equation as $f$, which reflects the fact that each component of $\mathbf{X}$ is a non-diffusive scalar associated with the fluid particle that $\mathbf{X}$ serves to label. In principle, $f$ could be determined by solving Eq. (7) for $\mathbf{G}$ and combining the result with Eq. (16), but it would be easier to simply solve Eq. (17) for $f$ directly.

It is instructive to consider the situation in which the velocity field is itself a non-diffusive scalar (or more precisely a non-diffusive vector). In this case Eq. (17) becomes $\partial \mathbf{u} / \partial t+\mathbf{u} \cdot \nabla \mathbf{u}=0$, which is the inviscid Burgers' equation in three space dimensions. In spite of its nonlinearity, this equation describes the free propagation of noninteracting fluid particles. Equation (15) then becomes $\mathbf{u}(\mathbf{x}, t)=\mathbf{u}_{0}(\mathbf{X})$, which combines with Eq. (4) to determine the Lagrangian trajectories in the form $\mathbf{x}=\mathbf{X}+t \mathbf{u}_{0}(\mathbf{X})=\mathbf{X}+t \mathbf{u}(\mathbf{x}, t)$, so that $\mathbf{G}(t ; \mathbf{x})=\mathbf{x}-t \mathbf{u}(\mathbf{x}, t)$. Equation (16) then becomes $\mathbf{u}(\mathbf{x}, t)$ $=\mathbf{u}_{0}(\mathbf{x}-t \mathbf{u}(\mathbf{x}, t))$, which is the well known implicit solution to the inviscid Burgers' equation. ${ }^{5}$

To recapitulate, if the velocity field is sufficiently simple that $\mathbf{G}(t ; \mathbf{x})$ can be obtained in closed form, either by solving Eq. (4) to obtain $\mathbf{F}$ and solving Eq. (5) for $\mathbf{X}$, or in the separable case by solving Eq. (9) to obtain $\mathbf{F}_{s}$ and obtaining $\mathbf{G}_{s}$ from Eq. (12), then the solution to Eq. (17) may be immediately obtained from Eq. (16). This exact solution is valid for an arbitrary initial non-diffusive scalar field $f_{0}(\mathbf{x})$ at $t=0$.

\section{CONSERVED DENSITIES}

A conserved quantity is defined to be a quantity which is neither created nor destroyed and locally moves with velocity u. In nonrelativistic physics, the quintessential conserved quantity is mass. A conserved density $\rho(\mathbf{x}, t)$ is the amount of a conserved quantity per unit volume at $(\mathbf{x}, t)$. Because conserved quantities move with velocity $\mathbf{u}$, the total amount of a conserved quantity within any Lagrangian volume $V(t)$ is constant in time, so that

$$
\int_{V(t)} d \mathbf{x} \rho(\mathbf{x}, t)=\int_{V(0)} d \mathbf{X} \rho_{0}(\mathbf{X}),
$$

where $\rho_{0}(\mathbf{x}) \equiv \rho(\mathbf{x}, 0)$ is the initial density field, and $d \mathbf{x}$ and $d \mathbf{X}$ denote the volume elements in the coordinates $\mathbf{x}$ and $\mathbf{X}$, respectively. By using Eq. (6) to transform the integration variable in the integral on the right from $\mathbf{X}$ to $\mathbf{x}$, we obtain

$$
\int_{V(t)} d \mathbf{x} \rho(\mathbf{x}, t)=\int_{V(t)} d \mathbf{x} J(\mathbf{x}, t) \rho_{0}(\mathbf{G}(t ; \mathbf{x})),
$$

where $J(\mathbf{x}, t) \equiv\|\nabla \mathbf{G}\|$ is the Jacobian of the transformation; that is, the absolute value of the determinant of $\nabla \mathbf{G}$. Clearly, $J(\mathbf{x}, 0)=1$. Because the region $V(t)$ is arbitrary, we may conclude that

$$
\rho(\mathbf{x}, t)=J(\mathbf{x}, t) \rho_{0}(\mathbf{G}(t ; \mathbf{x})),
$$

which provides an explicit solution for any conserved density $\rho(\mathbf{x}, t)$ in terms of its initial condition $\rho_{0}(\mathbf{x})$ and the function $\mathbf{G}$ (which also determines $J$ ). The special case in which $\rho_{0}(\mathbf{x})=1$ shows that $J(\mathbf{x}, t)$ is itself a conserved density. 
Equation (20) can be expressed in an alternative form that is less convenient for actual evaluation but is often more convenient theoretically. We introduce the identity $\int d \mathbf{X} \delta(\mathbf{X}-\mathbf{G})=1$ into Eq. (20) to obtain

$$
\rho(\mathbf{x}, t)=\int d \mathbf{X} \rho_{0}(\mathbf{X}) J(\mathbf{x}, t) \delta(\mathbf{X}-\mathbf{G}(t ; \mathbf{x})),
$$

where $\delta(\mathbf{x})$ is the Dirac delta function. The quantity $J(\mathbf{x}, t)$ $\delta(\mathbf{X}-\mathbf{G}(t ; \mathbf{x}))$ vanishes unless $\mathbf{x}=\mathbf{F}(t ; \mathbf{X})$, and its integral over all $\mathbf{x}$ has the finite value $\int d \mathbf{x} J(\mathbf{x}, t) \delta(\mathbf{X}$ $-\mathbf{G}(t ; \mathbf{x}))=\int d \mathbf{X} \delta(\mathbf{X}-\mathbf{G})=1$. It follows that $J(\mathbf{x}, t)$ $\delta(\mathbf{X}-\mathbf{G}(t ; \mathbf{x}))=\delta(\mathbf{x}-\mathbf{F}(t ; \mathbf{X}))$, so that Eq. (21) becomes

$$
\rho(\mathbf{x}, t)=\int d \mathbf{X} \rho_{0}(\mathbf{X}) \delta(\mathbf{x}-\mathbf{F}(t ; \mathbf{X})) .
$$

We remark that Eq. (22) also applies to nonequilibrium ensemble densities in statistical physics and is frequently useful in that context. ${ }^{6}$

By differentiating Eq. (22) with respect to time at constant $\mathbf{x}$, we find that $\rho(\mathbf{x}, t)$ satisfies the PDE

$$
\frac{\partial \rho}{\partial t}+\nabla \cdot(\rho \mathbf{u})=0
$$

which is the usual continuity equation for a conserved density. Because all conserved densities satisfy the same PDE, Eq. (23), different conserved densities differ only in their initial conditions. In particular, $J$ is a conserved density so it too satisfies the same equation,

$$
\frac{\partial J}{\partial t}+\nabla \cdot(J \mathbf{u})=0
$$

Moreover, because $J$ is determined entirely by the Lagrangian trajectories, it is the same for any conserved density, and it follows immediately from Eqs. (20) and (16) that the ratio of any two conserved densities is a non-diffusive scalar, and hence satisfies Eq. (17). Thus, $\rho / J$ is a non-diffusive scalar, which is also apparent from Eq. (20).

We have shown that any conserved density is determined in terms of its initial conditions by Eq. (20), and that any such density also satisfies Eq. (23). Conversely, Eq. (20) provides the exact solution of the continuity Eq. (23) for an arbitrary initial density field $\rho_{0}(\mathbf{x})$, which is the main point of interest in the present context. This solution is formally valid in general, but is useful only when the velocity field is sufficiently simple that the Lagrangian trajectories are integrable.

\section{LAGRANGIAN SURFACES}

A Lagrangian surface $S(t)$ is a surface made up of Lagrangian points satisfying Eq. (4), so that each point $\mathbf{x}$ on the surface moves with the local fluid velocity $\mathbf{u}(\mathbf{x}, t)$. A Lagrangian surface is therefore the set of all points $\mathbf{x}$ that satisfy Eq. (5), where $\mathbf{X}$ lies on the initial surface $S(0)$. The initial surface may be specified in various ways. Perhaps the most basic is by an expression of the form ${ }^{7}$

$$
\mathbf{X}=\mathbf{Q}_{0}(\alpha, \beta),
$$

where $\mathbf{Q}_{0}(\alpha, \beta)$ is parameterized in such a way that it sweeps over $S(0)$ as the parameters $(\alpha, \beta)$ are varied. For example, a sphere of radius $R$ may be defined in terms of spherical coordinates $(r, \theta, \phi)$ by letting $(\alpha, \beta)=(\theta, \phi)$ and setting $\mathbf{Q}_{0}(\alpha, \beta)=\operatorname{R} \mathbf{e}_{r}(\theta, \phi)$, where $\mathbf{e}_{r}(\theta, \phi)$ is the unit vector in the radial direction. In this description, the Lagrangian surface $S(t)$ that evolves from an initial surface $S(0)$ is defined by substituting Eq. (25) into Eq. (5) to obtain

$$
\mathbf{x}=\mathbf{Q}(t ; \alpha, \beta),
$$

where

$$
\mathbf{Q}(t ; \alpha, \beta)=\mathbf{F}\left(t ; \mathbf{Q}_{0}(\alpha, \beta)\right),
$$

and $\mathbf{Q}(0 ; \alpha, \beta)=\mathbf{Q}_{0}(\alpha, \beta)$. Once the initial Lagrangian surface at $t=0$ has been suitably parameterized by Eq. (25), its paramaterization at any later time is given by Eq. (26), in which $\mathbf{Q}$ is determined in terms of $\mathbf{F}$ by Eq. (27). Thus, we again obtain an exact solution in terms of the Lagrangian trajectories.

An alternative description of a Lagrangian surface is obtained when the points $\mathbf{X}$ on the initial surface $S(0)$ are defined by

$$
f_{0}(\mathbf{X})=0 \text {. }
$$

It then follows from Eq. (15) that the non-diffusive scalar $f(\mathbf{x}, t)$ defined by Eq. (16) vanishes on $S(t)$, so that $S(t)$ is determined by the equation

$$
f(\mathbf{x}, t)=0 .
$$

In this description, the exact solution for Lagrangian interface motion is given by Eq. (29), with $f(\mathbf{x}, t)$ given by Eq. (16). Thus, there is an intimate relation between Lagrangian surfaces and non-diffusive scalars, and any smooth nondiffusive scalar that takes on zero values may be used to define a Lagrangian surface via Eq. (29). This correspondence is not unique, because there is an uncountable infinity of non-diffusive scalars for which Eq. (29) defines the same $S(t)$. In particular, if $h=M(f+g)-M(g)$, where $M(x)$ is monotonic and $g$ is an arbitrary smooth function, then the equations $f=0$ and $h=0$ both define the same surface.

\section{EXAMPLES}

We have shown that when the velocity field is sufficiently simple that Eq. (4) can be explicitly solved in closed form to obtain the Lagrangian particle trajectories, as described by the functions $\mathbf{F}$ and $\mathbf{G}$, these functions immediately determine exact analytical solutions to Eq. (17) for non-diffusive scalars and Eq. (23) for conserved densities, as well as for Lagrangian surfaces in the form of Eq. (26) or Eq. (29). We emphasize that for the given velocity field, these solutions are the general solutions valid for arbitrary initial conditions. In contrast to the velocity field, these initial conditions need not be simple or symmetrical, and because the solutions are further distorted by the velocity field as they evolve, they tend to become even more complicated as time proceeds. The various relations we have derived can be used to generate a variety of complicated asymmetrical and fully threedimensional exact solutions to these classes of problems. In this section, we shall illustrate the application of these results by means of examples.

The examples to be considered are drawn from the broad class of radial velocity fields in spherical coordinates. They 
were not selected for their relevance to problems of real physical interest, but rather to illustrate the high degree of complexity that can readily be accommodated and tractably treated by the formalism that we have discussed. These examples are also peripherally related to the important physical problem of analyzing interfacial instabilities in inertial confinement fusion. ${ }^{8,9}$ The resulting analytical solutions describe the purely kinematical aspects of the time evolution of Lagrangian interfaces in spherical geometry. The dynamical evolution and stability of such interfaces are much more complicated, because they must evolve in such a way as to preserve pressure continuity, and this condition perturbs the velocity field. The kinematical effects are still present, but they become entangled or intermingled with dynamical effects, thereby making it difficult to separate the two. Results of the present type may be useful in this connection, because they contain only kinematics and can therefore serve as a basis for comparison with dynamical stability analyses in which both types of effects are present simultaneously. 8,9

We begin with the particularly simple example of an incompressible spherically symmetric radial velocity field in spherical coordinates; that is, $\mathbf{u}=u(r, t) \mathbf{e}_{r}$, where $\boldsymbol{\nabla} \cdot \mathbf{u}=r^{-2}$ $\partial\left(r^{2} u\right) / \partial r=0$. The latter equation can immediately be integrated to yield $u(r, t)=a(t) / r^{2}$, where $a(t)$ is an arbitrary function of time. The velocity field is therefore separable, but this example is so simple that the machinery of Sec. III is not really needed. Equation (4) now becomes

$$
\dot{\mathbf{x}}=\frac{d\left(r \mathbf{e}_{r}\right)}{d t}=\dot{r} \mathbf{e}_{r}+r \dot{\mathbf{e}}_{r}=\frac{a(t)}{r^{2}} \mathbf{e}_{r} .
$$

The identity $\mathbf{e}_{r} \cdot \mathbf{e}_{r}=1$ implies that $\dot{\mathbf{e}}_{r} \cdot \mathbf{e}_{r}=0$, so the dot product of $\mathbf{e}_{r}$ with Eq. (30) reduces to

$$
\dot{r}=\frac{a(t)}{r^{2}} .
$$

Equations (30) and (31) combine to imply that $\dot{\mathbf{e}}_{r}=0$, so that $\theta=\dot{\phi}=0$ and $(\theta, \phi)=\left(\theta_{0}, \phi_{0}\right)$, where $\theta_{0}$ and $\phi_{0}$ are the angular coordinates of the initial point $\mathbf{X}$.

The function $a(t)$ may be eliminated in terms of the time dependence of any particular reference Lagrangian radius $R(t)$ satisfying Eq. (31), for which $\dot{R}=a(t) / R^{2}$, so that $a(t)=R^{2} \dot{R}$. In problems involving a nearly spherical interface between two materials, which are ubiquitous in inertial confinement fusion, it is natural to define $R(t)$ as the radius of the unperturbed interface. Equation (31) then becomes $\dot{r}=R^{2} \dot{R} / r^{2}$, which integrates to $r^{3}-r_{0}^{3}=R^{3}-R_{0}^{3}$, where $R_{0} \equiv R(0)$. Thus,

$$
r=\left[R^{3}(t)-R_{0}^{3}+r_{0}^{3}\right]^{1 / 3},
$$

and conversely

$$
r_{0}=\left[R_{0}^{3}-R^{3}(t)+r^{3}\right]^{1 / 3},
$$

where $r_{0}$ is the radial coordinate of $\mathbf{X}$.

According to Eq. (16), a non-diffusive scalar function with initial value $f_{0}(r, \theta, \phi)$ at $t=0$ is given at any later time $t$ by

$$
f(r, \theta, \phi, t)=f_{0}\left(\left[R_{0}^{3}-R^{3}(t)+r^{3}\right]^{1 / 3}, \theta, \phi\right),
$$

which is therefore the exact general solution of Eq. (17) for the particular velocity field under consideration. Similarly, according to Eqs. (26) and (27), a Lagrangian surface initially defined by $r=Q_{0}(\theta, \phi)$ at $t=0$ is given at any later time by

$$
r=\left[R^{3}(t)-R_{0}^{3}+Q_{0}^{3}(\theta, \phi)\right]^{1 / 3} .
$$

If we wish to regard this surface as a Lagrangian deformation of the spherical surface $r=R(t)$, we must require these two surfaces to enclose the same volume. Because they are both Lagrangian, this condition will be satisfied for all times if it is satisfied initially, which requires that we define $R_{0}$ by

$$
\begin{aligned}
\frac{4 \pi}{3} R_{0}^{3} & =\int \sin \theta d \theta d \phi \int_{0}^{Q_{0}} r^{2} d r \\
& =\frac{1}{3} \int \sin \theta d \theta d \phi Q_{0}^{3}(\theta, \phi) .
\end{aligned}
$$

A somewhat more interesting class of examples is obtained by removing the condition of incompressibility. Consider, for example, the spherically symmetric radial velocity field $\mathbf{u}(\mathbf{x}, t)=U_{0} e^{-k r} \cos \omega t \mathbf{e}_{r}$, which is of the separable form considered in Sec. III with $\tau=\omega^{-1} \sin \omega t$ and $\mathbf{U}(\mathbf{x})=U_{0} e^{-k r} \mathbf{e}_{r}$. In this case, Eq. (9) becomes

$$
\frac{d \mathbf{x}}{d \tau}=\frac{d r}{d \tau} \mathbf{e}_{r}+r \frac{d \mathbf{e}_{r}}{d \tau}=U_{0} e^{-k r} \mathbf{e}_{r},
$$

from which it follows as before that $d \mathbf{e}_{r} / d \tau=0$, so that $d \theta / d \tau=d \phi / d \tau=0$. Thus, $(\theta, \phi)=\left(\theta_{0}, \phi_{0}\right) \quad$ and $\quad d r / d \tau$ $=U_{0} e^{-k r}$, which can be integrated to yield $e^{k r}=e_{0}^{k r}+k U_{0} \tau$. This equation implies that

$$
r=\frac{1}{k} \ln \left(e^{k r_{0}}+k U_{0} \tau\right),
$$

and conversely

$$
r_{0}=\frac{1}{k} \ln \left(e^{k r}-k U_{0} \tau\right) .
$$

According to Eq. (16), a non-diffusive scalar function with the initial value $f_{0}(r, \theta, \phi)$ at $t=0$ is given at any later time $t$ by

$$
f(r, \theta, \phi, t)=f_{0}\left(k^{-1} \ln \mu(r, t), \theta, \phi\right),
$$

where $\mu(r, t) \equiv e^{k r}-\left(k U_{0} / \omega\right) \sin \omega t$. Equation (40) is therefore the exact general solution of Eq. (17) for the particular velocity field under consideration. Similarly, according to Eqs. (26) and (27) a Lagrangian surface initially defined by $r=Q_{0}(\theta, \phi)$ at $t=0$ is given at any later time by

$$
r=\frac{1}{k} \ln \left(e^{k Q_{0}(\theta, \phi)}+k U_{0} \omega^{-1} \sin \omega t\right) .
$$

To obtain the corresponding solution for a conserved density, we must evaluate the determinant $J(\mathbf{x}, t)=\|\nabla \mathbf{G}\|$. Because $\mathbf{X}=r_{0} \mathbf{e}_{r}\left(\theta_{0}, \phi_{0}\right)$, this evaluation could be accomplished by combining Eqs. (6) and (39) to determine $\mathbf{G}$ and then evaluating $\nabla \mathbf{G}$ and taking its determinant. In the present context, however, it is simpler to observe that $J$ is the ratio of the volume elements in the $\mathbf{X}$ and $\mathbf{x}$ coordinates, and because $(\theta, \phi)=\left(\theta_{0}, \phi_{0}\right)$ this ratio reduces to $J=\left(r_{0} / r\right)^{2}\left|\partial r_{0} / \partial r\right|$, which can be evaluated from Eq. (39). The result is 


$$
J(\mathbf{x}, t)=J(r, t)=\frac{e^{k r}[\ln \mu(r, t)]^{2}}{k^{2} r^{2}|\mu(r, t)|} .
$$

According to Eq. (20), a conserved density with the initial value $\rho_{0}(r, \theta, \phi)$ at $t=0$ is given at any later time $t$ by

$$
\rho(r, \theta, \phi, t)=\frac{e^{k r}[\ln \mu(r, t)]^{2}}{k^{2} r^{2}|\mu(r, t)|} \rho_{0}\left(k^{-1} \ln \mu(r, t), \theta, \phi\right) .
$$

The exact general solution of the continuity Eq. (23) for this particular velocity field is therefore given by Eq. (43), which again exhibits complicated nonlinearities.

The preceding development can be extended to separable radial velocity fields that are neither incompressible nor spherically symmetric, for which $\mathbf{U}(\mathbf{x})$ takes the form

$$
\mathbf{U}(\mathbf{x})=U(r, \theta, \phi) \mathbf{e}_{r},
$$

in which the radial velocity has an arbitrary angular dependence on $(\theta, \phi)$. Equation (9) then becomes

$$
\frac{d \mathbf{x}}{d \tau}=\frac{d r}{d \tau} \mathbf{e}_{r}+r \frac{d \mathbf{e}_{r}}{d \tau}=U(r, \theta, \phi) \mathbf{e}_{r},
$$

from which it again follows that $d \mathbf{e}_{r} / d \tau=0$, so that $d \theta / d \tau$ $=d \phi / d \tau=0$. Thus, $(\theta, \phi)=\left(\theta_{0}, \phi_{0}\right)$ and $d r / d \tau=U(r, \theta, \phi)$, which can be integrated at constant $(\theta, \phi)$ to yield

$$
\int_{r_{0}}^{r} \frac{d r^{\prime}}{U\left(r^{\prime}, \theta_{0}, \phi_{0}\right)}=\tau
$$

Equation (46) implicitly determines $r=F_{s}\left(\tau ; r_{0}, \theta_{0}, \phi_{0}\right)$ and conversely $r_{0}=F_{s}(-\tau ; r, \theta, \phi)$, and hence the functions $\mathbf{F}(t ; \mathbf{X})=F_{s}\left(\tau(t) ; r_{0}, \theta_{0}, \phi_{0}\right) \mathbf{e}_{r}\left(\theta_{0}, \phi_{0}\right)$ and $\mathbf{G}(t ; \mathbf{x})=F_{s}(-\tau(t) ; r, \theta$, $\phi) \mathbf{e}_{r}(\theta, \phi)$. These functions can be explicitly determined if the integral in Eq. (46) can be evaluated in closed form and solved for $r$. When this is the case, the relations given in Secs. IV-VI provide exact solutions for non-diffusive scalars, conserved densities, and Lagrangian interfaces for the particular velocity field of interest.

\section{DISCUSSION}

We have shown how the equivalence between nonlinear ODEs and linear PDEs can be exploited to construct a wide variety of asymmetric and fully three-dimensional exact solutions for the kinematical time evolution of non-diffusive scalars, conserved densities, and Lagrangian surfaces advected by an arbitrary fluid velocity field. These solutions can be expressed in closed form if the velocity field is sufficiently simple that the trajectories of the Lagrangian fluid particles are integrable. The present treatment complements the converse development of Smith, ${ }^{2}$ and it is hoped that it will serve to further foster, promote, and disseminate an awareness of and facility with the techniques involved. It also provides a unified framework for systematically constructing particular exact solutions of the present type. Advection and interface dynamics are essential ingredients in computational fluid dynamics, so it is also hoped that this formalism will be found useful in verifying the multidimensional numerical methods and computer codes used to solve practical problems in fluid dynamics.

\section{ACKNOWLEDGMENTS}

The author is grateful to Rod Douglass for thoughtful comments on the manuscript, and to Peter Amendt, Karnig Mikaelian, and Bill Moran for helpful discussions and comments on an earlier related manuscript. The author also thanks the anonymous reviewers for suggestions that resulted in significant improvements. A portion of this work was performed under the auspices of the U. S. Department of Energy by the University of California Lawrence Livermore National Laboratory under Contract No. W-7405-Eng-48.

${ }^{1}$ C. Carathéodory, Calculus of Variations and Partial Differential Equations of the First Order, 2nd English ed. (Chelsea, New York, 1982).

${ }^{2}$ B. R. Smith, Jr., "First-order partial differential equations in classical dynamics," Am. J. Phys. 77, 1147-1153 (2009).

${ }^{3}$ J. D. Ramshaw, Elements of Computational Fluid Dynamics (Imperial College Press/World Scientific, London/Singapore, 2011).

${ }^{4}$ L. E. Malvern, Introduction to the Mechanics of a Continuous Medium (Prentice Hall, Englewood Cliffs, NJ, 1969).

${ }^{5}$ G. B. Whitham, Linear and Nonlinear Waves (John Wiley \& Sons, New York, 1974)

${ }^{6}$ J. D. Ramshaw, "Remarks on non-Hamiltonian statistical mechanics," Europhys. Lett. 59, 319-323 (2002).

${ }^{7}$ G. A. Korn and T. M. Korn, Mathematical Handbook for Scientists and Engineers, 2nd ed. (McGraw-Hill, New York, 1961).

${ }^{8}$ P. Amendt, J. D. Colvin, J. D. Ramshaw, H. F. Robey, and O. L. Landen, "Modified Bell-Plesset effect with compressibility: Application to doubleshell ignition target designs," Phys. Plasmas 10, 820-829 (2003).

${ }^{9}$ J. D. Ramshaw and P. A. Amendt, "Linearized stability analysis of accelerated planar and spherical fluid interfaces with slow compression," Phys. Rev. E 67, 056304-1-5 (2003). 